\title{
Selected film and video distribution sources
}

\author{
Anthea Kennedy \\ 57 Ravensworth Road \\ London NW10 5NP \\ UK \\ Email: Anthea.Kennedy@btopenworld.com \\ British Film Institute \\ 21 Stephen Street \\ London W1T 1LN \\ UK \\ Tel.: 02079578935 \\ Fax: 02075805830 \\ Email: bookings.films@bfi.org.uk \\ Web: www.bfi.org.uk \\ Electronic Arts Intermix \\ 535 West 22nd Street, 5th Floor \\ New York, NY 10011-1119 \\ USA \\ Tel.: +1 2123370694 \\ Fax: +1 2123370679 \\ Email: info@eai.org \\ Web: www.eai.org/eai/ \\ Lux \\ 18 Shacklewell Lane, \\ London E8 2EZ, \\ UK \\ Tel.: +44 (0)20 75033980 \\ Email: mike.sperlinger@lux.org.uk \\ Web: www.lux.org.uk
}


Scott Rankin

Illinois State University

College of Fine Arts

Campus Box 5600

Normal, IL 61790-5600

USA

Tel.: +1 3094388090

Email: sdranki@ilstu.edu

Web: www.cfa.ilstu.edu/sdranki/

Video Data Bank

112 S. Michigan Avenue

Chicago, IL 60603

USA

Tel.: +1 3123453550

Fax: +13125418073

Email: info@vdb.org

Web: www.vdb.org 